\title{
Diabetic versus non-diabetic colour vision after cataract surgery
}

Line Kessel, Anita Alsing, Michael Larsen

\begin{abstract}
Aims-To examine whether the colour vision abnormalities found in phakic patients with diabetes mellitus is preserved after removal of the lens by cataract surgery.

Methods-21 diabetic (16 IDDM and five NIDDM) and 19 non-diabetic patients of comparable age, postoperative visual acuity, and sex distribution, all aphakic or pseudophakic following cataract surgery, had their monocular colour vision examined using the Farnsworth-Munsell 100 hue test. The fundus status of the diabetic patients ranged from no retinopathy to photocoagulation treated proliferative diabetic retinopathy. Patients with macular oedema were specifically excluded from the study.

Results-The error scores of both the diabetic (mean 146 (SD 94)) and the nondiabetic patients $(83$ (79)) did not deviate significantly from the age related normal range. The error score in the diabetic group was significantly higher than in the non-diabetic group $(p=0.02)$ but the amplitude of the difference was small in comparison with previous studies of phakic subjects. The error scores in the diabetic group were not correlated with the degree of retinopathy $(\mathrm{p}>0.2)$.

Conclusion-After cataract surgery only a minor difference exists between the colour vision scores of diabetic and non-diabetic patients. This indicates that accelerated yellowing of the lens in diabetes is the predominant cause of the colour vision anomaly found in phakic diabetic patients.

(Br f Ophthalmol 1999;83:1042-1045)
\end{abstract}

Since the first report of a case of severe acquired dyschromatopsia in a patient with diabetes mellitus ${ }^{1}$ several studies have examined the relation between diabetic retinopathy and colour vision. ${ }^{23}$ The colour vision defect often precedes the onset of retinopathy. Consequently it has been suggested that dyschromatopsia may indicate which diabetic patients are likely to develop diabetic retinopathy. ${ }^{2-4}$ A prospective study of colour vision testing was unsuccessful in detecting early diabetic retinopathy, however.

Yellow chromophores are continuously accumulated inside the lens as a consequence of physiological aging. This increasingly dense colour filter primarily reduces the transmission of blue light to the retina. The normal age related changes in colour vision test perform- ance as well as the changes seen in diabetic patients are predominantly in the blue-yellow colour vision axis. ${ }^{2411}$ This is in theoretical agreement with the optical effects of an increasingly dense yellow/brown optical filter.

Non-enzymatic glycation of lens proteins is a likely cause of the browning of the lens. ${ }^{56}$ This spontaneous protein denaturation process is accelerated in diabetes because the glucose concentration is elevated in all tissues that do not depend on insulin for glucose uptake. ${ }^{78}$ The heterogeneous mixture of brownish advanced glycation products includes species that are fluorescent. In humans, lens autofluorescence appears to increase in proportion to lens browning. $^{58}$

The working hypothesis of the present study is that the colour vision anomaly seen in diabetic patients is caused predominantly or exclusively by accelerated aging of the lens. To assess colour vision in diabetes independently of the colour filtering effects of the lens, we have compared the colour vision of diabetic and non-diabetic patients after removal of the lens by cataract surgery.

\section{Subjects}

All patients were recruited among patients who had undergone cataract surgery at our institution no more than 4 years and no less than 4 months before the initiation of the study. We examined 21 diabetic patients (16 with insulin dependent diabetes (IDDM) and five with non-insulin dependent diabetes (NIDDM)) and 19 non-diabetic patients. The two groups were of comparable age, postoperative visual acuity, and sex distribution (Table 1). Exclusion criteria were secondary cataract, glaucoma, and diabetic macular oedema within two optic disc diameters of the foveola. Patients with diabetic macular oedema were excluded because this condition is associated with a specific dysfunction of foveal vision. ${ }^{9}$ The study was approved by the local medical ethics committee. All participants gave their informed consent according to the Helsinki Declaration.

Table 1 Clinical data of the non-diabetic and diabetic patients

\begin{tabular}{lll}
\hline & Non-diabetics & Diabetics \\
\hline Number & 19 & 21 \\
Sex, female (male) & $10(9)$ & $10(11)$ \\
Age in years & $57(33-75)$ & $55(25-71)$ \\
Snellen visual acuity & $0.7(0.3-1)^{\star}$ & $0.7(0.3-1)^{\star}$ \\
No of pseudophakic/aphakic & $18 / 1$ & $13 / 8$ \\
Retinopathy & & $2(0-4)^{\star}$ \\
HbA & $(\%)$ & $8.4(6.3-10.8)$ \\
Duration of diabetes (years) & & $29(8-57)$ \\
No of IDDM/NIDDM & & $16 / 5$
\end{tabular}

Values in mean (range) except ${ }^{\star}$ in median (range). $\mathrm{HbA}_{1 \mathrm{c}}$ wa unavailable in four patients. $\mathrm{HbA}_{1 \mathrm{c}}$ normal range: $4.1-6.4$. 


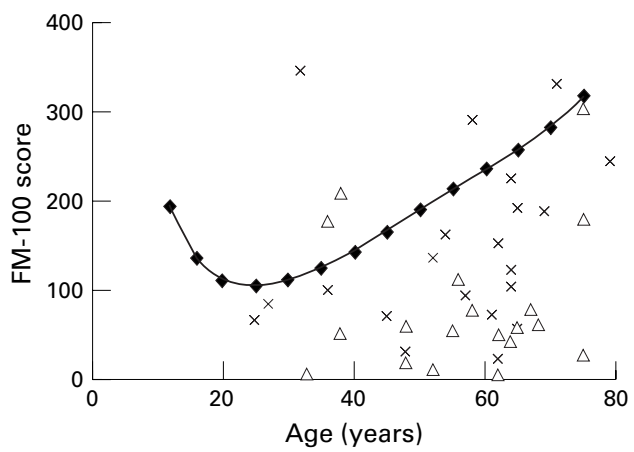

Figure 1 Scatter plot of Farnsworth-Munsell 100 hue colour vision error scores in aphakic or pseudophakic diabetic patients (crosses) and aphakic or pseudophakic non-diabetic patients (triangles). The upper normal range for phakic subjects (mean $+2 S D$ ) is also shown according to Verriest ${ }^{11}$ (diamonds).

\section{Methods}

Colour vision was tested using the FarnsworthMunsell 100 hue test under illumination with a Macbeth Easel lamp. None of the patients had any previous experience with the test. Only one eye was examined for each patient. If both eyes had been operated on, the eye with the best visual acuity was chosen. If no difference existed the right eye was chosen. The retina was examined using slit lamp biomicroscopy and fundus photography. Retinopathy was classified as follows: grade 0 indicates no retinopathy (four patients); grade 1 includes microaneurysms and/or haemorrhages smaller than one arteriolar width in diameter (three patients); grade 2 includes larger haemorrhages, cotton wool spots, and hard exudates (six patients); grade 3 includes non-fibrotic proliferative diabetic retinopathy (three patients); grade 4 includes fibrovascular proliferations (five patients). Six patients had received photocoagulation treatment for proliferative diabetic retinopathy in the examined eye. Metabolic control was assessed by the most recent $\mathrm{HbA}_{1 \mathrm{c}}$ value. The fraction of glycated haemoglobin $\left(\mathrm{HbA}_{1 \mathrm{c}}\right)$ serves as a blood glucose level index that covers the preceding 3 months (normal range $4.1-6.4 \%$ ).

The Spearman correlation coefficient was used to assess the correlation between error score and duration of diabetes, $\mathrm{HbA}_{1 \mathrm{c}}$, and age. The correlation between error score and

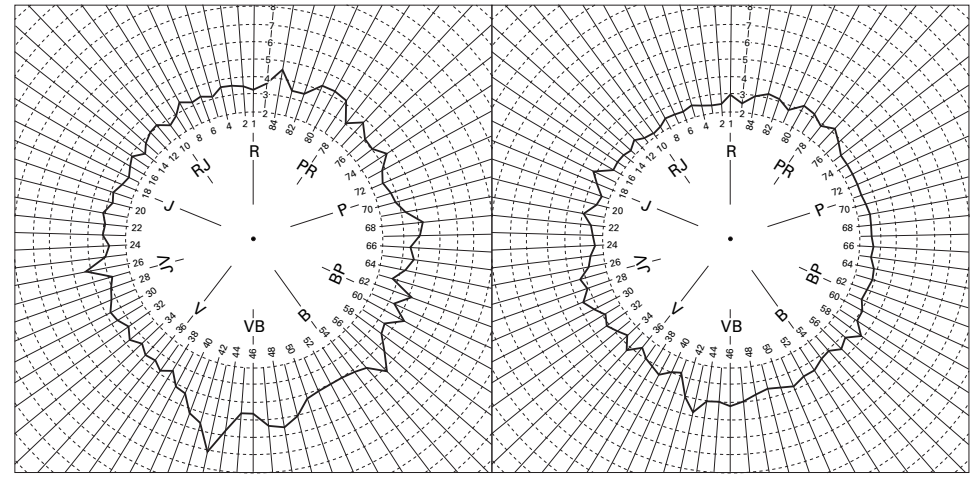

Figure 2 Farnsworth-Munsell 100 colour vision error scores in standard diagrammatic presentation. Mean scores for the 21 aphakic or pseudophakic diabetic patients (left) and the 19 aphakic or psedudophakic non-diabetic patients (right). In both groups a significant preponderance of errors is found in the blue-yellow axis $(p=0.001$ for the diabetic group and $p=0.002$ for the non-diabetic group). degree of retinopathy or visual acuity was evaluated using the Spearman rank correlation coefficient. $\mathrm{p}$ Values were calculated using the two tailed $t$ test and 0.05 as the level of statistical significance. The distribution of error scores in relation to the reference interval for healthy phakic subjects was evaluated using the Mann-Whitney-Wilcoxon test.

The spectral distribution of the FM-100 hue error score was assessed by dividing the standard FM-100 error score plot into four quadrants. ${ }^{2}$ Quadrant I includes caps $75-11$; quadrant II caps 12-32; quadrant III caps 33-53 and quadrant IV caps 54-74. The error sum of quadrants I and III is divided by the error sum of quadrants II and IV. When the result is greater than 1 , a tritan axis is said to be present, and the greater the ratio the more pronounced the tritan axis defect. The predominance of errors along this axis is labelled tritan because it is a characteristic of patients who demonstrate a tritan defect on Ishihara testing. ${ }^{10}$

\section{Results}

The Farnsworth-Munsell 100 hue error scores, both for the diabetic patients (mean 146 (SD 94)) and for the non-diabetic patients (83 (79)), did not deviate significantly from the age related normal distribution for phakic nondiabetic subjects (Fig 1). ${ }^{11}$ No significant correlation was found between the FM-100 score and $\mathrm{HbA}_{1 \mathrm{c}}$, age, duration of diabetes, grade of retinopathy, or postoperative visual acuity $(p>0.2)$. Both groups showed a significant tritan colour vision axis $(p=0.001$ for the diabetic group and $\mathrm{p}=0.002$ for the nondiabetic group; Fig 2). The error scores were significantly higher in the diabetic than in the non-diabetic patients $(p=0.02)$ but the difference was smaller than previously found in phakic subjects. $^{249}$

\section{Discussion}

In the present study of colour vision after removal of the lens by cataract surgery, we compared diabetic patients with various degrees of retinopathy and non-diabetic patients. We did not find significantly abnormal error scores in any of the two groups. This is in sharp contrast with the results found in phakic diabetic patients, of whom a large proportion have markedly elevated error scores compared with healthy phakic subjects. ${ }^{249}$

In some phakic studies of colour vision in diabetic patients a difference between patients with and without retinopathy was found ${ }^{42}$ whereas others have failed to show such a difference. ${ }^{9}{ }^{13}$ Only two studies have demonstrated a significant correlation between the degree of retinopathy and the degree of colour vision defect. ${ }^{2}{ }^{4}$ In the present study we found no correlation between diabetic retinopathy and colour vision error score.

The colour vision changes associated with aging and diabetes in phakic patients are found in the blue-yellow axis of the FM-100 test. ${ }^{2}$ This may theoretically be attributed to the gradual age related yellowing of the lens that is known to be accelerated in diabetes. ${ }^{8}$ The yel- 


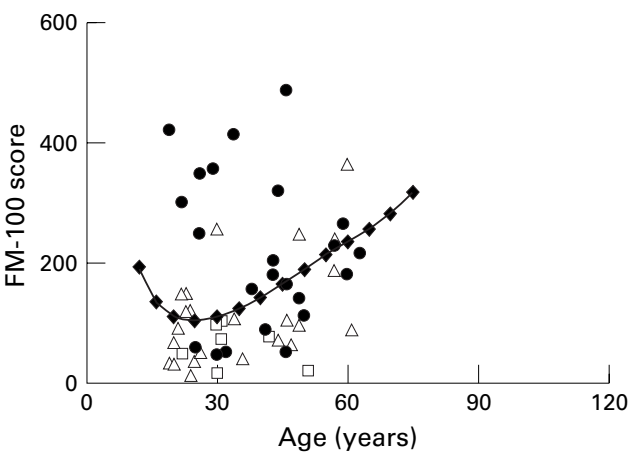

Figure 3 Farnsworth-Munsell 100 colour vision error scores in relation to chronological age in a group of phakic diabetic patients examined by Bresnick et al (excluding subjects with macular oedema). Markers indicate patients with no diabetic retinopathy (squares), microaneurisms and exudates (triangles), and proliferative retinopathy (solid circles). The upper normal range is also shown (mean +2 $S D)$ according to Verriest ${ }^{11}$ (diamonds).

low hue is produced by preferential absorption of the blue component of white light upon transillumination of the lens. Accelerated yellowing, rather than neuronal or vascular changes in the retina, thus appears to be the primary cause of the colour vision anomaly observed in diabetic patients. This is supported by the lack of a convincing correlation between the colour vision defects and the onset of diabetic retinopathy. ${ }^{313}$

The effect of lens aging on colour vision in phakic diabetic patients may be evaluated theoretically by calculating an equivalent healthy lens age for each individual diabetic patient on the basis of chronological age and duration of diabetes. Colour vision data can be found in a previous study of patients with diabetes mellitus by Bresnick et $a l^{2}$ and the relation between age, duration of diabetes, and lens fluorescence can be estimated according to Bleeker et $a l^{8}$ and Larsen ${ }^{14}$ :

$$
A_{\text {eqv }}=\left(7,98^{\star} A+8,78^{\star} D-33.9\right) / 6.36
$$

where $A_{e q v}$ is the equivalent lens age for healthy subjects - that is, the age at which a non-diabetic subject is expected to have the same lens fluorescence as a diabetic patient with a specific chronological age $A$ and duration $D$ of diabetes. ${ }^{8}{ }^{14}$ Plotting the colour vision error scores against the reference curve for healthy subjects ${ }^{11}$ before (Fig 3) and after (Fig 4) transformation of chronological age to equivalent healthy lens age demonstrates that accelerated lens aging appears to account for most of the difference in colour vision between the diabetic patients and the healthy subjects. A few diabetic patients with proliferative retinopathy remain above the normal range, but these patients would probably fall within the normal range if their lens ages were corrected by direct fluorometry instead of by using statistical average estimates for lens fluorescence. ${ }^{7}$

Both the development of diabetic retinopathy and the accelerated aging of the lens are related to poor metabolic control. ${ }^{715}$ It is therefore likely that patients with advanced retinopathy and poor colour vision have been in particularly poor metabolic control and con-

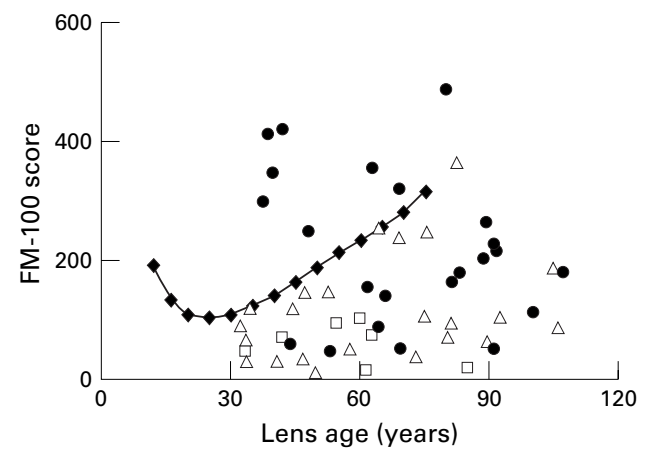

Figure 4 Farnsworth-Munsell 100 hue colour vision error scores in a group of phakic diabetic patients examined by Bresnick et al (same patients as in Fig 3) shown in relation to the apparent lens age calculated by transforming chronological age and diabetes duration to equivalent healthy lens age to account for the accelerated accumulation of brown chromophores in the diabetic lens. ${ }^{814}$ Markers indicate patients with no diabetic retinopathy (squares), microaneurisms and exudates (triangles), and proliferative retinopathy (solid circles). The upper normal range is also shown (mean $+2 S D$ ) according to Verriest ${ }^{11}$ (diamonds).

sequently have browner lenses than patients with mild retinopathy.

In agreement with this, a previous study of colour vision where young phakic diabetic patients were found to have a high proportion of anomalies in comparison with healthy patients of the same age demonstrated the difference to be less pronounced if the optical density of the lens was accounted for by calculation of an equivalent healthy lens age in a manner similar to that described above. ${ }^{16}$

In conclusion, both the diabetic and the non-diabetic group in the present study had colour vision error scores within the normal age related range. This supports the theory that colour vision defects in diabetes are caused primarily by accelerated lens aging. The moderately higher error score in the diabetic compared with the non-diabetic group that remains after removal of the lens may indeed be caused by a true element of retinal or optic nerve dysfunction in diabetes. This should be studied in phakic subjects after correction for the optical filtering effect of the lens by lens fluorometry.

The study was supported by the Novo Nordisk Foundation and the Danish Association for the Prevention of Blindness and Eye Diseases (Værn om Synet).

1 Dubois-Poulsen A, Cochet P. Un cas de dyschromatopsie diabetique. Bull Soc Ophthalmol 1954;4:323-30.

2 Bresnick GH, Condit RS, Palta M, et al. Association of hue Bresnick GH, Condit RS, Palta M, et al. Association of hue
discrimation loss and diabetic retinopathy. Arch Ophthalmol

3 Aspinall PA, Kinnear PR, Duncan LJ, et al. Prediction of diabetic retinopathy from clinical variables and colour vision data. Diabetes Care 1983;6:144-8.

4 Kinnear PR, Aspinall PA, Lakowski R. The diabetic eye and colour vision. Trans Ophthalmol Soc UK 1972;92:69-78.

5 Monnier VM, Kohn RR, Cerami A. Accelerated age-related browning of human collagen in diabetes mellitus. Proc Natl Acad Sci 1984;61:583-7.

6 Stevens VJ, Rouzer CA, Monnier VM, et al. Diabetic cataract formation: potential role of glycosylation of lens crystallins. Proc Natl Acad Sci 1978;75:2918-22.

7 Kjer B, Larsen M, Bendtson I, et al. Lens autofluorescence in diabetes compared with the level of glycosylated hemoglobin A $_{1 \mathrm{c}}$. Acta Ophthalmol 1987;65:100-2.

8 Bleeker JC, van Best JA, Vrij L, et al. Autofluorescence of the lens in diabetic and healthy subjects by fluorophotometry. Invest Ophthalmol Vis Sci 1986;27:791-4.

9 Trick GL, Burde RM, Gordon MO, et al. The relationship between hue discrimination and contrast sensitivity deficits in patients with diabetes mellitus. Ophthalmology 1988;95: 
10 Farnsworth D. The Farnsworth-Munsell 100-hue and dichotomous tests for color vision. F Opt Soc Am 1943;33: dichotom $568-78$.

11 Verriest G, van Laethem J, Uvijls A. A new assessment of the normal ranges of the Farnsworth-Munsell 100-hue test scores. Am $\mathcal{F}$ Ophthalmol 1982;93:635-42

12 Knowles PJ, Tregear SJ, Ripley LG, et al. Colour vision in diabetic and normal pseudophakes is worse than expected. Eye 1996;10:113-6.

13 Moloney J, Drury MI. Retinopathy and retinal function in insulin-dependent diabetes mellitus. Br f Ophthalmol 1982; 62:759-61.
14 Larsen M. Fluorometric assessment of equivalent lens age for diabetics (letter to the editor). Invest Ophthalmol Vis Sci 1993;34:2607.

15 The Diabetes Control and Complications Trial Research Group. The effect of intensive treatment of diabetes on the development and progression of long-term complications in insulin-dependent diabetes mellitus. N Engl F Med 1993; 329:977-86.

16 Hardy KJ, Scarpello JHB, Foster DH, et al. Effect of diabetes associated increases in lens optical density on colour discrimination in insulin dependent diabetes. $\mathrm{Br} f$ Ophthalmol 1994;78:754-6. 